\title{
Evaluation of the Effectiveness of Antibiotics against Eosinophilic Pustular Folliculitis
}

\author{
Sachiko Ono ${ }^{a}$ Yosuke Yamamoto $^{b}$ Atsushi Otsuka ${ }^{a}$ Kenji Kabashima ${ }^{a}$ \\ Yoshiki Miyachi $^{a}$ \\ Departments of ${ }^{\mathrm{a}}$ Dermatology and ${ }^{\mathrm{b}}$ Healthcare Epidemiology, Kyoto University Graduate \\ School of Medicine, Kyoto, Japan
}

\section{Key Words}

Eosinophilic pustular folliculitis · Therapeutic options · Antibiotics · Tetracyclines · Macrolides

\begin{abstract}
Eosinophilic pustular folliculitis (EPF) is a chronic intractable pruritic dermatosis. Although indomethacin is generally effective against EPF and considered as a first-line therapy, quite a few patients with indomethacin still suffer from the symptoms. Among other therapeutic options, some antibiotics have been reported to be effective; however, there has been no epidemiological description regarding oral antibiotics use in patients with EPF. In this study, we investigated the frequency of antibiotics use and the effectiveness in patients with EPF.
\end{abstract}

Eosinophilic pustular folliculitis (EPF) is a chronic intractable pruritic dermatosis that is characterized by massive eosinophilic infiltrates involving the pilosebaceous units. The diagnosis is made in patients showing repeated pruritic follicular papules and sterile pustules arranged in arcuate plaques with central healing and peripheral spread with the histopathological finding of folliculotropic infiltration of eosinophils. The cause of EPF has not been elucidated, but it has been suggested that it may be caused by hypersensitivity reactions to various infections, medications and autoimmune disorders that are facilitated by sebaceous gland lipid-derived eosinophilic chemotactic and activation factors [1]. Indomethacin is generally effective against EPF, and cyclooxygenase metabolites are assumed to be involved in the pathomechanism of EPF [2]. In particular, we have previously reported that prostaglandin $\mathrm{D}_{2}$ directly attracts inflammatory cells such as T helper type 2 (Th2) cells and also induces a marked upregulation of eotaxin-3 in sebocytes, which may 
explain the massive eosinophil infiltration around pilosebaceous units [3]. Other therapeutic options, including nonsteroidal anti-inflammatory drug derivatives, oral and topical corticosteroids, dapsone, cyclosporine, ultraviolet phototherapy, retinoid, antihistamines, antifungals, scabicides and some oral antibiotics, have also been used to treat patients with EPF $[1,2,4,5]$. Several case reports have revealed that among oral antibiotics, tetracyclines such as minocycline and doxycycline and macrolides such as roxithromycin were effective against EPF [4, 6]. However, there has been no epidemiological description regarding the use of oral antibiotics in patients with EPF. Therefore, we investigated the frequency of antibiotic use and its effectiveness in patients with EPF. In the present study, we collected detailed information about current antibiotic use and asked attending doctors about their perceived effectiveness in each patient. The antibiotics were assessed using a 3-point ordinal scale as follows: not effective/unknown, moderately effective and highly effective.

The study was based on case report forms of 143 outpatients with EPF who visited dermatology clinics in 67 hospitals across Japan between 2010 and 2011. Participation was limited to patients with the diagnosis of EPF made by dermatologists in each facility with the help of the following criteria: having lesions characterized by erythematous plaques with itchy follicular papulopustules, histopathologically manifested by folliculocentric infiltrations of eosinophils. Of those patients, $34(23.8 \%)$ were treated with oral antibiotics, either minocycline (MINO; $\mathrm{n}=19$ ), doxycycline (DOXY; $\mathrm{n}=3$ ), tetracycline (TC; $\mathrm{n}=1$ ), roxithromycin (RXM; $n=10$ ) or cefcapene pivoxil hydrochloride hydrate (CFPN-PI; $n=1$ ). The baseline characteristics of the patients are shown in table 1. It is notable that antibiotics were moderately or highly effective in $79.41 \%$ of the patients (table 2). RXM was evaluated as moderately or highly effective in $90 \%$ of the patients who were prescribed the drug. In contrast, the present results revealed that MINO was moderately or highly effective in $73.6 \%$ of the patients. Regarding other tetracyclines, DOXY and TC were reported to be moderately effective in all the patients who were prescribed these drugs.

It is assumed that the effectiveness of tetracyclines against EPF is due to their antibacterial and anti-inflammatory properties [4]. These drugs modify the fatty acid balance by increasing the concentration of esterified fatty acids and decreasing the concentration of free fatty acids, which are considered to be the obligatory substratum for the production of inflammatory mediators and exert chemotactic effects on eosinophils [4]. In addition, tetracyclines markedly depress the migration of human leukocytes in vitro and accelerate neutrophil apoptosis, limiting the ability of neutrophils to damage the surrounding tissues in patients with EPF [4].

The results suggest that RXM is also effective against EPF and that it might be worthwhile to use RXM in patients with EPF who are nonresponsive to indomethacin or MINO. RXM suppresses the production of Th2 chemokines [7]. The evidence accumulated to date indicates that Th2-mediated immunologic mechanisms are involved in EPF pathogenesis [8], which may explain the reason why RXM is also effective against EPF. Because both tetracyclines and macrolides are considered to be largely effective due to their antiinflammatory properties as mentioned previously, they might be effective against EPF even at concentrations less than their minimal inhibitory concentrations.

The present study has several limitations. Although participation was limited to patients who had visited hospitals in recent years, the results might include incorrect patient information. Because the therapeutic response was assessed only by each attending doctor, it was often associated with a measurement bias. Therefore, we asked doctors in each facility to evaluate the therapeutic response once they had reviewed the medical records. 
Although indomethacin is the primary treatment for EPF, further investigation is necessary to identify other options. In case indomethacin is ineffective, we believe that antibiotics, particularly tetracyclines and macrolides, might be effective in EPF treatment.

\section{Acknowledgements}

This study was supported in part by a grant-in-aid from the Ministry of Health, Labour and Welfare of Japan.

\section{Disclosure Statement}

The authors have no financial interests to declare.

\section{References}

1 Nervi SJ, Schwartz RA, Dmochowski M: Eosinophilic pustular folliculitis: a 40 year retrospect. J Am Acad Dermatol 2006;55:285-289.

- Fukamachi S, Kabashima K, Sugita K, Kobayashi M, Tokura Y: Therapeutic effectiveness of various treatments for eosinophilic pustular folliculitis. Acta Derm Venereol 2009;89:155-159.

-3 Nakahigashi K, Doi H, Otsua A, Hirabayashi T, Murakami M, Urade Y, Tanizaki H, Egawa G, Miyachi Y, Kabashima K: PGD2 induces eotaxin-3 via PPARgamma from sebocytes: a possible pathogenesis of eosinophilic pustular folliculitis. J Allergy Clin Immunol 2012;129:536-543.

4 Brazzelli V, Barbagallo T, Prestinari F, Ciocca O, Vassallo C, Borroni G: HIV seronegative eosinophilic pustular folliculitis successfully treated with doxicycline. J Eur Acad Dermatol Venerol 2004;18:467-470.

5 Ellis E, Scheinfeld N: Eosinophilic pustular folliculitis: a comprehensive review of treatment options. Am J Clin Dermatol 2004;5:189-197.

6 McCalmont TH, Altemus D, Maurer T, Berger TG: Eosinophilic folliculitis. The histologic spectrum. Am J Dermatopathol 1995;17:439-446.

7 Kobayashi M, Shimauchi T, Hino R, Tokura Y: Roxithromycin downmodulates Th2 chemokine production by keratinocytes and chemokine receptor expression on Th2 cells: its dual inhibitory effects on the ligands and the receptors. Cell Immunol 2004;228:27-33.

8 Otsuka A, Doi H, Miyachi Y, Kabashima K: Treatment of eosinophilic pustular folliculitis with cyclosporin: suppression of mRNA expression of IL-4 and IL-13. J Eur Acad Dermatol Venerol 2010;24:1489-1491. 
Ono et al.: Evaluation of the Effectiveness of Antibiotics against Eosinophilic Pustular Folliculitis

Table 1. Baseline characteristics of the subjects

\begin{tabular}{lc}
\hline & $\begin{array}{c}\text { EPF with oral antibiotics } \\
(\mathrm{n}=34)\end{array}$ \\
\hline Age, years & $44.1 \pm 16.4$ \\
Disease duration, years & $2.1 \pm 3.1$ \\
Treatment duration, months & $19.4 \pm 49.1$ \\
Male & 58.8 \\
Oral indomethacin use & 58.8 \\
Oral corticosteroid use & 0 \\
Oral cyclosporine use & 0 \\
Topical indomethacin use & 23.5 \\
Topical corticosteroid use & 58.8 \\
Topical tacrolimus use & 20.6 \\
Topical antifungal agent use & 2.9 \\
Topical antibiotic use & 11.8 \\
HIV infection & 11.8 \\
\hline Values represent mean \pm standard deviation or percentage.
\end{tabular}

Table 2. Effectiveness of antibiotics

\begin{tabular}{lccc}
\hline & $\begin{array}{l}\text { Not effective/ } \\
\text { unknown }\end{array}$ & $\begin{array}{l}\text { Moderately } \\
\text { effective }\end{array}$ & Highly effective \\
\hline MINO, \% & $26.32(5 / 19)$ & $57.89(11 / 19)$ & $15.79(3 / 19)$ \\
DOXY, \% & $0(0 / 3)$ & $100(3 / 3)$ & $0(0 / 3)$ \\
RXM, \% & $10(1 / 10)$ & $80(8 / 10)$ & $10(1 / 10)$ \\
TC, \% & $0(0 / 1)$ & $100(1 / 1)$ & $0(0 / 1)$ \\
CFPN-PI, \% & $100(1 / 1)$ & $0(0 / 1)$ & $0(0 / 1)$ \\
Total, \% & $20.59(7)$ & $67.65(23)$ & $11.76(4)$ \\
\hline
\end{tabular}

Figures in parentheses are absolute numbers. 